\title{
Erratum to: Prognostic significance of telomere maintenance mechanisms in pediatric high-grade gliomas
}

\author{
Kathleen Dorris • Matthew Sobo $\cdot$ Arzu Onar-Thomas • Eshini Panditharatna \\ Charles B. Stevenson - Sharon L. Gardner • Mariko D. DeWire • \\ Christopher R. Pierson • Randal Olshefski • Sandra A. Rempel · \\ Stewart Goldman · Lili Miles · Maryam Fouladi $\cdot$ Rachid Drissi
}

Published online: 28 June 2014

(c) Springer Science+Business Media New York 2014

\section{J Neurooncol (2014) 117:67-76}

DOI 10.1007/s11060-014-1374-9

In Table 2 of the original publication, the data in table columns 6-13 were incorrectly displayed for the table rows of IDs 37, 45-47. The correct data are shown in bold below.

The online version of the original article can be found under doi:10.1007/s11060-014-1374-9.

K. Dorris - M. Sobo - E. Panditharatna .

M. D. DeWire · M. Fouladi · R. Drissi ( $\square)$

Division of Oncology, Cancer and Blood Diseases Institute, Cincinnati Children's Hospital Medical Center, MLC 7013,

3333 Burnet Ave, Cincinnati, OH 45229, USA

e-mail: rachid.drissi@cchmc.org

\section{A. Onar-Thomas}

Department of Biostatistics, St. Jude Children's Research

Hospital, 262 Danny Thomas Place, Memphis, TN 38105, USA

C. B. Stevenson

Division of Pediatric Neurosurgery, Cincinnati Children's Hospital Medical Center, 3333 Burnet Ave, Cincinnati, OH 45229, USA

\section{S. L. Gardner}

Department of Pediatric Hematology/Oncology, New York University Langone Medical Center, 160 E. 32nd St., New York, NY 10016, USA

C. R. Pierson

Department of Pathology \& Laboratory Medicine, Nationwide Children's Hospital, 700 Children's Dr., Columbus, OH 43205, USA
R. Olshefski

Division of Hematology/Oncology, Nationwide Children's Hospital, 700 Children's Dr., Columbus, OH 43205, USA

S. A. Rempel

Barbara Jane Levy Laboratory of Molecular Neuro-Oncology, Department of Neurosurgery, and Josephine Ford Cancer Institute, Hermelin Brain Tumor Center, Henry Ford Hospital, 2799 West Grand Blvd., Detroit, MI 48202, USA

\section{S. Goldman}

Department of Pediatric Hematology/Oncology/Bone Marrow Transplantation, Ann \& Robert H. Lurie Children's Hospital of Chicago, 225 E. Chicago Avenue, Chicago, IL 60611, USA

\section{Miles}

Division of Pathology and Laboratory Medicine, Cincinnati

Children's Hospital Medical Center, 3333 Burnet Ave., Cincinnati, OH 45229, USA 
Table 2 Summary of clinical characteristics and telomere maintenance assay results

\begin{tabular}{|c|c|c|c|c|c|c|c|c|c|c|c|c|}
\hline ID & Gender & $\begin{array}{l}\text { Age } \\
\text { (years) }\end{array}$ & Tumor & Pathology & $\begin{array}{l}\text { Specimen } \\
\text { Timing }\end{array}$ & Resection & $\begin{array}{l}\text { Telomerase } \\
\text { activity by TRAP }\end{array}$ & ALT & $\begin{array}{l}\text { TERT } \\
\text { (RQ) }\end{array}$ & $\begin{array}{l}\text { TERC } \\
\text { (RQ) }\end{array}$ & $\begin{array}{l}\text { Time to death or last } \\
\text { follow-up (years) }\end{array}$ & Deceased \\
\hline 1 & M & 8.8 & DIPG & GBM & A & N/A & - & + & 5.38 & 8.56 & 0.84 & $\mathrm{Y}$ \\
\hline 2 & $\mathrm{~F}$ & 12.1 & DIPG & $\mathrm{AA}$ & $\mathrm{D}$ & Partial & N/A & N/A & 52.29 & 1.46 & 2.26 & $\mathrm{Y}$ \\
\hline 3 & F & 22.2 & HGG & GBM & $\mathrm{D}$ & Partial & - & + & 0 & 0.31 & 1.53 & $\mathrm{~N}$ \\
\hline 4 & F & 12.0 & DIPG & $\mathrm{AA}$ & A & N/A & - & + & 0.50 & 5.03 & 1.21 & $\mathrm{Y}$ \\
\hline 5 & F & 10.2 & HGG & AA & $\mathrm{D}$ & Partial & - & + & 0.11 & 0.23 & 11.19 & $\mathrm{~N}$ \\
\hline 6 & $\mathrm{~F}$ & 17.9 & HGG & GBM & $\mathrm{R}$ & Partial & N/A & N/A & 0.49 & 1.58 & 3.47 & $\mathrm{Y}$ \\
\hline 7 & M & 17.9 & HGG & AA & $\mathrm{D}$ & Partial & + & N/A & 80.21 & 1.40 & 1.18 & Y \\
\hline 8 & $\mathrm{~F}$ & 2.5 & HGG & AA & $\mathrm{D}$ & Total & - & N/A & 0 & 4.031 & 5.36 & $\mathrm{~N}$ \\
\hline $9^{\#}$ & M & 17.9 & HGG & $\mathrm{AA}$ & $\mathrm{D}$ & Partial & - & + & 0 & 0.84 & 2.98 & $\mathrm{Y}$ \\
\hline $10^{\#}$ & M & 17.9 & HGG & GBM & $\mathrm{R}$ & Partial & + & + & 4.058 & 0.32 & 2.98 & $\mathrm{Y}$ \\
\hline 11 & M & 5.9 & HGG & GBM & $\mathrm{D}$ & Partial & N/A & N/A & 0.44 & 1.70 & 2 & $\mathrm{~N}$ \\
\hline 12 & M & 13.8 & HGG & $\mathrm{AA}$ & $\mathrm{D}$ & Total & N/A & N/A & 0.36 & 1.18 & 0.27 & $\mathrm{~N}$ \\
\hline 13 & M & 12.1 & HGG & GBM & $\mathrm{D}$ & Total & N/A & N/A & 0 & 0.43 & 3.23 & $\mathrm{~N}$ \\
\hline 14 & M & 3.7 & HGG & $\mathrm{AA}$ & $\mathrm{D}$ & Partial & - & N/A & 0.14 & 0.21 & 0.26 & $\mathrm{~N}$ \\
\hline $15^{*}$ & $\mathrm{M}$ & 12.1 & HGG & GBM & $\mathrm{R}$ & Total & N/A & N/A & 57.91 & 5.29 & 3.23 & $\mathrm{~N}$ \\
\hline $16^{*}$ & $\mathrm{M}$ & 12.1 & HGG & GBM & $\mathrm{R}$ & Total & N/A & N/A & 71.41 & 1.73 & 3.23 & $\mathrm{~N}$ \\
\hline $17^{\wedge}$ & $\mathrm{F}$ & 0.02 & HGG & GBM & $\mathrm{R}$ & Partial & - & N/A & N/A & N/A & 21.38 & $\mathrm{~N}$ \\
\hline 21 & F & 5.1 & DIPG & GBM & A & N/A & - & N/A & 3.58 & 5.22 & 1.16 & $\mathrm{Y}$ \\
\hline 22 & $\mathrm{~F}$ & 3.2 & DIPG & AA & A & N/A & N/A & N/A & 5.44 & 26.016 & 0.56 & Y \\
\hline 23 & M & 5.4 & DIPG & GBM & A & N/A & + & - & 6.37 & 2.47 & 0.68 & $\mathrm{Y}$ \\
\hline 24 & $\mathrm{~F}$ & 12.5 & DIPG & GBM & A & N/A & + & - & 4.83 & 6.40 & 0.81 & $\mathrm{Y}$ \\
\hline 25 & $\mathrm{~F}$ & 12.1 & DIPG & GBM & A & N/A & N/A & N/A & 0.80 & 2.38 & 0.78 & Y \\
\hline 26 & M & 2.8 & DIPG & GBM & A & N/A & - & - & 1.68 & 2.45 & 2.64 & Y \\
\hline 27 & $\mathrm{~F}$ & 3.4 & DIPG & GBM & A & N/A & - & - & 0.12 & 0.53 & 0.42 & $\mathrm{Y}$ \\
\hline 36 & F & 4.7 & DIPG & GBM & A & N/A & - & - & 9.027 & 2.65 & 1.58 & $\mathrm{Y}$ \\
\hline 37 & $\mathbf{F}$ & 4.8 & DIPG & GBM & $\mathbf{A}$ & N/A & - & - & 3.92 & 5.82 & 1.25 & $\mathbf{Y}$ \\
\hline 45 & $\mathbf{F}$ & 9.6 & DIPG & GBM & $\mathbf{A}$ & N/A & - & + & 15.22 & 5.92 & 0.64 & $\mathbf{Y}$ \\
\hline 46 & $\mathbf{F}$ & 4.2 & DIPG & GBM & $\mathbf{A}$ & N/A & - & - & 0.14 & 0.84 & 0.81 & $\mathbf{Y}$ \\
\hline 47 & $\mathbf{F}$ & 4.6 & DIPG & GBM & $\mathbf{A}$ & N/A & + & - & 7.12 & 2.85 & 1.95 & $\mathbf{Y}$ \\
\hline 28 & F & 6.9 & HGG & GBM & $\mathrm{R}$ & Total & + & - & 5.31 & 1.056 & 3.58 & $\mathrm{Y}$ \\
\hline 29 & $\mathrm{~F}$ & 20 & HGG & GBM & $\mathrm{R}$ & Partial & - & + & 0.33 & 0.18 & 0.83 & $\mathrm{~N}$ \\
\hline 30 & $\mathrm{M}$ & 6 & HGG & GBM & $\mathrm{D}$ & Partial & - & N/A & 8.45 & 19.36 & 0.08 & $\mathrm{Y}$ \\
\hline 31 & $\mathrm{~F}$ & 15.8 & HGG & AA & $\mathrm{D}$ & Partial & - & - & 0.30 & 1.44 & 2.25 & $\mathrm{~N}$ \\
\hline 32 & $\mathrm{~F}$ & 13.8 & HGG & GBM & $\mathrm{D}$ & Total & + & - & 499.71 & 0.20 & 1 & $\mathrm{Y}$ \\
\hline 33 & $\mathrm{M}$ & 15.2 & HGG & GBM & $\mathrm{D}$ & Partial & + & - & 2.99 & 3.21 & 1.42 & $\mathrm{Y}$ \\
\hline 34 & $\mathrm{~F}$ & 7.4 & HGG & AA & $\mathrm{D}$ & Partial & - & + & 0 & 3.13 & 1.58 & $\mathrm{Y}$ \\
\hline 35 & $\mathrm{M}$ & 15.2 & HGG & GBM & $\mathrm{D}$ & Total & + & - & 15.46 & 0.15 & 3 & $\mathrm{~N}$ \\
\hline 38 & $\mathrm{M}$ & 17.8 & HGG & $\mathrm{AA}$ & $\mathrm{R}$ & Partial & - & + & 0.087 & 0.62 & 1.5 & $\mathrm{~N}$ \\
\hline $39^{+}$ & M & 19.2 & HGG & GBM & $\mathrm{D}$ & Total & - & + & 1.18 & 0.42 & 4.58 & $\mathrm{~N}$ \\
\hline $40^{+}$ & M & 19.2 & HGG & GBM & $\mathrm{R}$ & Total & - & - & 0.30 & 0.20 & 4.58 & $\mathrm{~N}$ \\
\hline 41 & $\mathrm{M}$ & 16.6 & HGG & GBM & $\mathrm{D}$ & Partial & - & N/A & 0.30 & 0.41 & 0.65 & $\mathrm{Y}$ \\
\hline 42 & $\mathrm{M}$ & 8.4 & HGG & GBM & $\mathrm{D}$ & Partial & - & - & 0.23 & 0.76 & 0.8 & Y \\
\hline 43 & $\mathrm{~F}$ & 11.6 & HGG & GBM & $\mathrm{D}$ & Partial & - & + & 1.83 & 8.20 & 0.77 & $\mathrm{Y}$ \\
\hline 44 & $\mathrm{M}$ & 12.4 & HGG & GBM & $\mathrm{D}$ & Partial & + & - & 19.80 & 0.70 & 1.87 & $\mathrm{Y}$ \\
\hline 48 & M & 15 & HGG & GBM & $\mathrm{D}$ & Total & + & - & 6.65 & 0.84 & 0.91 & $\mathrm{Y}$ \\
\hline 49 & $\mathrm{M}$ & 12 & HGG & GBM & $\mathrm{R}$ & Total & - & + & 4.33 & 0.90 & 2.24 & Y \\
\hline 50 & $\mathrm{M}$ & 20 & HGG & GBM & $\mathrm{D}$ & Total & - & - & 0.37 & 1.12 & 0.45 & $\mathrm{Y}$ \\
\hline
\end{tabular}

$H G G$ non-brainstem high-grade glioma, $D I P G$ diffuse intrinsic pontine glioma, $G B M$ glioblastoma multiforme, $A A$ anaplastic astrocytoma, $R Q$ relative quantification, specimen timing, $A$ autopsy, $D$ diagnosis, $R$ relapse

$\#, \wedge, *,{ }^{+}$Serial specimens from same patient 